\title{
Say hello to Dr Warwick Dunn! Metabolomics' new Reviews Editor
}

\author{
Royston Goodacre ${ }^{1}$
}

Published online: 16 February 2016

(C) Springer Science+Business Media New York 2016

Metabolomics is very pleased to welcome Dr Warwick (Rick) Dunn as its new Reviews Editor.

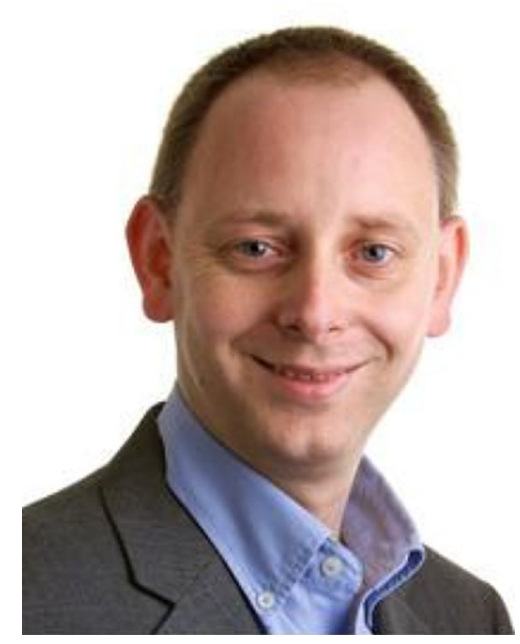

As the field of metabolomics continues to grow and expand there comes a time when it is apposite to summarise these burgeoning information in the form of reviews. Metabolomics has therefore decided to assign a dedicated reviews editor to handle all manuscripts that are submitted to the journal as review articles, and I am delighted that Rick has been appointed to this position.

Rick has an excellent track record in metabolomics and has published some key papers in mammalian

Royston Goodacre

Roy.Goodacre@manchester.ac.uk

1 School of Chemistry and Manchester Institute of Biotechnology, University of Manchester, 131 Princess Street, Manchester M1 7DN, UK metabolomics (including papers in this journal), has developed chromatography-mass spectrometry for largescale metabolomics (Dunn et al. 2011b)_which has been used to probe human metabolism (Dunn et al. 2005), as well as being part of the team involved in the metabolic network reconstructions of yeast and man (Herrgård et al. 2008; Thiele et al. 2013).

Rick has also published some first rate reviews. Including for example a comprehensive review on mammalian metabolomics published in Chemical Society Reviews (Dunn et al. 2011a), a community review of metabolite identification in this journal (Dunn et al. 2013), as well as insightful reviews in analytical journals detailing techniques that are being developed and employed for metabolomics (Dunn and Ellis 2005; Dunn et al. 2005). According to Thomas Reuter's Web of Science these reviews have average citation rates per year of well in excess of 31-39! It is therefore clear that Rick has a wealth of knowledge that means his appointment to Reviews Editor in this journal is very appropriate.

When I discussed this opportunity with Rick he was enthusiastic about this editorial position and said:

The field of metabolomics is growing at an exponential rate with new technological and biological developments leading to new scientific discoveries that impact on our daily lives in many different ways from our health through to the food we consume. As a journal we want to help our readers to keep in touch, in a single place, with recent developments and the impact they provide

The use of review articles to help new researchers in the field as well as to enhance the knowledge of scientists who have applied metabolomics for many years is an important process in our scientific 
education and dissemination of knowledge. At Metabolomics we want to provide a resource to allow this to occur

There are many types of reviews that Metabolomics would consider for publication, and these include: Critical Reviews - Tutorial Reviews - Mini-Reviews - Opinion Pieces. Moreover, this journal is not organism specific and so we welcome reviews in all discipline areas of metabolomics.

If you have suggestions for review topics or review articles then please contact Dr Warwick Dunn (w.dunn@bham.ac.uk) to discuss this further.

\section{References}

Dunn, W. B., \& Ellis, D. I. (2005). Metabolomics: Current analytical platforms and methodologies. TRAC-Trends in Analytical Chemistry, 24, 285-294.
Dunn, W. B., Bailey, N. J. C., \& Johnson, H. E. (2005). Measuring the metabolome: Current analytical technologies. Analyst, 130, 606-625.

Dunn, W. B., Broadhurst, D., Atherton, H. J., Goodacre, R., \& Griffin, J. L. (2011a). Systems level studies of mammalian metabolomes: The roles of mass spectrometry and nuclear magnetic resonance spectroscopy. Chemical Society Reviews, 40, 387-426.

Dunn, W. B., Broadhurst, D., Begley, P., Zelena, E., FrancisMcIntyre, S., Anderson, N., et al. (2011b). Procedures for largescale metabolic profiling of serum and plasma using gas chromatography and liquid chromatography coupled to mass spectrometry. Nature Protocols, 6, 1060-1083.

Dunn, W. B., Erban, A., Weber, R. J. M., Creek, D. J., Brown, M., Breitling, R., et al. (2013). Mass appeal: Metabolite identification in mass spectrometry-focused untargeted metabolomics. Metabolomics, 9, S44-S66.

Herrgård, M. J., Swainston, N., Dobson, P., Dunn, W. B., et al. (2008). A consensus yeast metabolic network obtained from a community approach to systems biology. Nature Biotechnology, $26,1155-1160$.

Thiele, I., Swainston, N., Fleming, R. M. T., Hoppe, A., Sahoo, S., et al. (2013). A community-driven global reconstruction of human metabolism. Nature Biotechnology, 31, 419-425. 